\title{
Comparison between the Hamiltonian Monte Carlo method and the Metropolis-Hastings method for coseismic fault model estimation
}

\section{Taisuke Yamada ( $\nabla$ taisuke.yamada.r3@dc.tohoku.ac.jp)}

Tohoku University Graduate School of Science Faculty of Science: Tohoku Daigaku Daigakuin Rigaku Kenkyuka Rigakubu https://orcid.org/0000-0002-0534-5396

\section{Keitaro Ohno}

Geospatial Information Authority of Japan

\section{Yusaku Ohta}

Tohoku University Graduate School of Science Faculty of Science: Tohoku Daigaku Daigakuin Rigaku Kenkyuka Rigakubu

\section{Research Article}

Keywords: Hamiltonian Monte Carlo, global navigation satellite system, Markov chain Monte Carlo, realtime GEONET Analysis system for rapid deformation, Bayesian inversion

Posted Date: February 24th, 2022

DOI: https://doi.org/10.21203/rs.3.rs-1368527/v1

License: (-) (i) This work is licensed under a Creative Commons Attribution 4.0 International License. Read Full License 


\section{Title page:}

\section{Full paper}

3 Comparison between the Hamiltonian Monte Carlo method and the Metropolis-

\section{Hastings method for coseismic fault model estimation}

5

6 Author \#1: Taisuke Yamada, Research Center for Prediction of Earthquakes and

7 Volcanic Eruptions, Graduate School of Science, Tohoku University, 6-6 Aza-Aoba,

8 Aramaki, Aoba-ku, Sendai 980-8578, Japan, taisuke.yamada.r3@dc.tohoku.ac.jp

9 Author \#2: Keitaro Ohno, Geospatial Information Authority of Japan, 1 Kitasato,

10 Tsukuba 305-0811, Japan, ohno-k96pi@mlit.go.jp

11 Author \#3: Yusaku Ohta, Research Center for Prediction of Earthquakes and Volcanic

12 Eruptions, Graduate School of Science, Tohoku University, 6-6 Aza-Aoba, Aramaki,

13 Aoba-ku, Sendai 980-8578, Japan, Division for the Establishment of Frontier Sciences

14 of Organization for Advanced Studies, Tohoku University, 2-1-1 Katahira, Aoba-

15 ku, Sendai 980-8577, Japan, International Research Institute of Disaster Science,

16 Tohoku University, Aoba 468-1, Aramaki, Aoba-ku, Sendai 980-8572, Japan, 
$17 \quad$ yusaku.ohta.d2@tohoku.ac.jp

18 Corresponding author: Taisuke Yamada

19 
20 Abstract

21 A rapid source fault estimation and quantitative assessment of the uncertainty of the

22 estimated model can elucidate the occurrence mechanism of earthquakes and inform

23 disaster damage mitigation. The Bayesian statistical method that addresses the posterior

24 distribution of unknowns is significant for uncertainty assessment. Particularly, the

25 Metropolis-Hastings (M-H) method, a kind of the Markov chain Monte Carlo (MCMC)

26 method, generally has many applications, including cosesimic fault estimation. However,

27 this method exhibits a trade-off between the transition distance and the acceptance ratio

28 of parameter transition candidates and requires a long mixing time, particularly in solving

29 high-dimensional problems. This necessitates a more efficient Bayesian method. In this

30 study, we developed a fault estimation algorithm using the Hamiltonian Monte Carlo

31 (HMC) method, which is generally considered to be more efficient than the other MCMC

32 method but not sufficiently feasible, to estimate the coseismic fault for the first time.

33 HMC can conduct sampling more intelligently with the gradient information of the

34 posterior distribution. We applied our algorithm to the 2016 Kumamoto earthquake $\left(\mathrm{M}_{\mathrm{j}}\right.$

35 7.3), and its sampling converged in $2 \times 10^{4}$ chains, including $1 \times 10^{3}$ burn-in chains. 
36 The estimated models satisfactorily accounted for the input data; the variance reduction

37 was approximately $88 \%$, and the estimated fault parameters and event magnitude were

38 consistent with those reported in previous studies. HMC could acquire similar results

39 using only $2 \%$ of the M-H chains. Moreover, the power spectral density (PSD) of each

40 model parameter's Markov chain showed that this method exhibited a low correlation

41 with the subsequent sample and a long transition distance per step. These results indicate

42 that $\mathrm{HMC}$ has advantages in terms of chain length than $\mathrm{M}-\mathrm{H}$, expecting a more efficient

43 estimation for a high-dimensional problem that requires a long mixing time or a problem

44 using non-linear Green's function, which has a large computational cost.

46 Keywords

47 Hamiltonian Monte Carlo, global navigation satellite system, Markov chain Monte Carlo,

48 real-time GEONET Analysis system for rapid deformation, Bayesian inversion

49

50 Main Text 


\section{1. Introduction}

52 To minimize earthquake and tsunami damage, the details of the coseismic fault must be

53 estimated rapidly. Surface displacement data supplied by the global navigation satellite

54 system (GNSS) can provide a stable estimation without underestimating the magnitude,

55 considering that the measurement of a seismometer can be saturated when a megathrust

56 occurs (e.g., Ohta et al. 2012). GNSS data are used in the real-time GEONET analysis

57 system for rapid deformation monitoring (REGARD) (Kawamoto et al. 2017), which was

58 jointly developed by the Geospatial Information Authority of Japan and Tohoku

59 University. This system analyzes a GNSS carrier phase in real-time to detect seismic

60 events and automatically and rapidly estimate the fault model. Fault models were

61 automatically estimated, for example, the 2016 Kumamoto earthquake (M JMA 7.3)

62 (Kawamoto et al. 2016), the 2019 Yamagata-Oki earthquake (MJMA 6.7), and the 2021

63 Fukushima-Oki earthquake (M MMA 7.3). These rapid inferences of REGARD include the

64 slip distribution model on a plate interface in a subduction zone and a single rectangular

65 fault estimation to address non-interplate earthquakes, such as inland and intra-slab

66 earthquakes. The fault models predicted by REGARD were also adopted as part of the 
67 real-time tsunami inundation and damage forecast system operated by the Cabinet Office,

68 Government of Japan (Musa et al. 2018; Ohta et al. 2018). In other words, it is important

69 to accurately understand the estimation uncertainty of the estimated fault models to

70 predict tsunamis and other natural disasters.

71 For the uncertainty evaluation, a method based on Bayesian statistics is generally used

72 to solve the inverse problem. This method is used to quantitatively assess the uncertainty

73 of the prediction using the posterior probability density function (PDF) of model

74 parameters while explicitly integrating prior information. Amey et al. (2018) applied the

75 Bayesian inversion method to the inference of the slip distribution in the 2014 Napa

76 Valley earthquake with self-similarity of the fault slip (Mai and Beroza 2002) as prior

77 information. In addition, many previous studies have used Bayesian inversion (e.g.,

78 Sambridge and Mosegaard 2002; Fukuda and Johnson 2008; Dettmer et al. 2014; Ohno

79 et al. 2022).

80 In the case of non-interplate earthquakes such as the 2019 Yamagata-Oki earthquake or

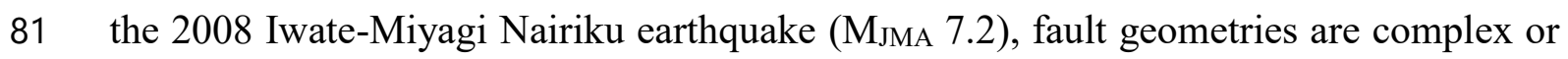

82 unknown, and many earthquakes are difficult to assume the fault geometry in advance 
83 (Ohta et al. 2008; Ohno et al. 2021). The assumption of fault geometry required in the

84 slip distribution model can make inferences ambiguous and affect the inferred result (e.g.,

85 Fukahata and Wright 2008; Fukuda and Johnson 2010; Duputel et al. 2014; Agata et al.

86 2021; Dutta et al. 2021).

87 There are some previous studies which are satisfied the two requirements above, rapid

88 estimation and quantitative uncertainty assessment; for example, Minson et al. (2014)

89 developed a real-time estimation method using Bayesian linear regression including the

90 fault geometry selection with the assessment of its uncertainty and the slip distribution

91 estimation on that geometry. Ohno et al. (2021) also developed Bayesian fault estimation

92 methods that acquire the uncertainty of the fault parameters of a single rectangular fault

93 by directly drawing the PDF using the Markov chain Monte Carlo (MCMC) method.

94 Their method incorporates parallel tempering (Swendsen and Wang 1986; Geyer 1991)

95 and an algorithm to automatically determine hyperparameters for real-time and fully

96 automatic fault estimation.

97 One of the MCMC sampling methods typically used in the context of Bayesian fault

98 estimation is the Metropolis-Hastings (M-H) method (Metropolis et al. 1953; Hastings 
1970). M-H forms a Markov chain based on the judgment of acceptance or rejection exhibits a disadvantageous trade-off relationship between the transition distance of the parameters and the acceptance ratio. In other words, M-H sampling is appropriate to decrease its acceptance ratio in proportion to the transition distance in the parameter space, especially for the sampling after the burn-in. Although the transition distance is often adjusted based on an acceptance ratio of approximately 30-50\%, this low acceptance makes the inference inefficient. Furthermore, with respect to the higher dimensional problems with vast parameter space, the acceptance ratio cannot be improved (the curse of dimensionality), and the transition distance is further shortened. To address the tradeoff problem, the M-H method requires a relatively long chain and mixing time.

112 Considering this problem, we focus on the Hamiltonian Monte Carlo (HMC; Duane et al. 1987) method instead of the M-H method. The HMC method requires a relatively short

114 Markov chain to converge a sampling because this method can transition long distances 
while maintaining an acceptance ratio that is nearly $100 \%$ by utilizing the gradient

116 information of the posterior PDF. Fichtner and Simute (2018) applied the weakly

117 nonlinear problem of source point determination and developed an efficient and accurate

118 algorithm. This study also proposed application to higher dimensional problems to

119 capitalize on the efficiency of HMC; in fact, HMC is frequently used in the study of

120 seismic tomography (e.g., Fichtner et al. 2019; Gebraad et al. 2020; Muir and Tkalcic

121 2020). Nevertheless, HMC has not been applied to fault estimation problems in the past,

122 and its applicability needs to be verified.

123 In this study, as an application test, we developed an algorithm for a single rectangular

124 fault estimation using HMC. To evaluate its performance, the algorithm was applied to

125 the 2016 Kumamoto earthquake. Furthermore, to examine the estimation accuracy and

126 efficiency of the HMC method, the results were compared with those of the conventional

127 M-H method. We mainly discuss the applicability of HMC to the estimation of earthquake

source fault models and briefly discuss the computational time for real-time usage. 


\section{Method}

130

\subsection{Bayes' theorem}

131

Bayesian inversion is based on Bayes' theorem (e.g., Tarantola 2005; Gelman et al.

2021) on a model parameter vector, $\boldsymbol{\theta}$, and a data vector, $\mathbf{d}$, used in estimation.

133

134

$$
f(\boldsymbol{\theta} \mid \mathbf{d})=\frac{f(\boldsymbol{\theta}) f(\mathbf{d} \mid \boldsymbol{\theta})}{f(\mathbf{d})}
$$

135

136

where $f(\boldsymbol{\theta} \mid \mathbf{d})$ is the posterior $\mathrm{PDF}, f(\boldsymbol{\theta})$ is the prior PDF, $f(\mathbf{d} \mid \boldsymbol{\theta})$ is the likelihood

137 function, and $f(\mathbf{d})$, which does not depend on the model parameters, is the

normalization constant. According to Equation (1), the posterior PDF is proportional to

the prior PDF and the likelihood function.

\subsection{Sampling method}

\subsubsection{Hamiltonian Monte Carlo method}

We developed a method for single rectangular fault estimation using the HMC method

(Duane et al. 1987; Neal 2011) and evaluated its ability in this study. First, in the HMC

method, an auxiliary parameter vector $\boldsymbol{p}$ with the same dimensions as the model 


$$
h(\boldsymbol{\theta})=-\log (f(\boldsymbol{\theta} \mid \mathbf{d}))
$$

152 is calculated using the set of $\boldsymbol{\theta}$ and $\boldsymbol{p}$. Subsequently, the transition in the phase space is

$$
p_{i}\left(t+\frac{1}{2}\right)=p_{i}(t)-\frac{\mathrm{e}}{2} \frac{\partial h(\boldsymbol{\theta}(t))}{\partial \theta_{i}}
$$

$$
\theta_{i}(t+1)=\theta_{i}(t)+\mathrm{e} p_{i}\left(t+\frac{1}{2}\right)
$$

$$
p_{i}(t+1)=p_{i}\left(t+\frac{1}{2}\right)-\frac{\mathrm{e}}{2} \frac{\partial h(\boldsymbol{\theta}(t+1))}{\partial \theta_{i}}
$$


162 where $\theta_{i}$ and $p_{i}$ are the $i$ th unknown and auxiliary parameters, respectively, $\mathrm{e}$ is the 163 step size, $t$ is the current step number, and L is the total step size. Equations (4)-(6) are 164 iterated from $t=1$ to $\mathrm{L}$, where $\mathrm{L}$ is the total step size of the leapfrog. Furthermore, e 165 and L are hyperparameters, and their initial settings are important for efficient estimation.

166 Subsequently, a numerical error, including the final parameters $\theta_{\mathrm{L}}$ and $p_{\mathrm{L}}$, is evaluated 167 using the acceptance ratio $p_{\text {accept }}$.

$$
r=\exp \left(H\left(\boldsymbol{\theta}^{(\tau)}, \boldsymbol{p}^{(\tau)}\right)-H\left(\boldsymbol{\theta}^{\left(\tau^{\prime}\right)}, \boldsymbol{p}^{\left(\tau^{\prime}\right)}\right)\right)
$$

174 the Hamiltonian is theoretically preserved, and only a small numerical error affects

175 Equation (8); therefore, $r$ is always close to 1, and a high probability of acceptance is 

is required to decrease the numerical error. and $\mathrm{L}$ are set. to $\boldsymbol{p}^{(\tau)}$.

3) Transition from the current point $\left(\boldsymbol{\theta}^{(\tau)}, \boldsymbol{p}^{(\tau)}\right)$ to the candidate point $\left(\boldsymbol{\theta}^{\left(\tau^{\prime}\right)}, \boldsymbol{p}^{\left(\tau^{\prime}\right)}\right)$ is performed using the leapfrog method and hyperparameters

2) Random numbers are generated from a standard normal distribution and set

4) The candidate point is accepted or rejected using the acceptance ratio 
HMC acts on the settings where the hyperparameters are $(e, L)=\left(10^{-3}, 10\right)$.

194

195

\subsubsection{No-U-Turn Sampler}

196

The HMC method requires hyperparameters e and L, which have a significant effect

197

on the sampling efficiency. Therefore, optimization is needed to use this method

efficiently. If $\mathrm{L}$ is too large, the leapfrog will generate "U-Turn" trajectories; therefore,

the final transition length can become shorter than the middle one. To solve this problem,

we employed the No-U-Turn Sampler (NUTS; Hoffman and Gelman 2014), the extended

201 method of the HMC.

202

While the usual HMC uses the constant L throughout the sampling, the NUTS does not

203

have the capability to set a constant $\mathrm{L}$ value. Instead, the leapfrog transition is terminated

204

based on the detection of "U-Turn". "U-Turn" in phase space defines the shortening of

205

distance $Q$ between a leftmost point, $\boldsymbol{\theta}^{+}$, and a rightmost point, $\boldsymbol{\theta}^{-}$, of leapfrog

206

transition, and then, it is determined using the time derivative of $Q$.

207

208

$$
Q=\frac{1}{2}\left(\boldsymbol{\theta}^{+}-\boldsymbol{\theta}^{-}\right)^{\mathrm{T}}\left(\boldsymbol{\theta}^{+}-\boldsymbol{\theta}^{-}\right)
$$




$$
\frac{d Q}{d \tau}=\left(\boldsymbol{\theta}^{+}-\boldsymbol{\theta}^{-}\right)^{\mathrm{T}} \boldsymbol{p}^{+}<0 \text { or }\left(\boldsymbol{\theta}^{+}-\boldsymbol{\theta}^{-}\right)^{\mathrm{T}} \boldsymbol{p}^{-}<0 \text { (10) }
$$

211 where the current sample number $\tau$ represents the time in the phase space, and

$212 \boldsymbol{p}^{+}$and $\boldsymbol{p}^{-}$are the momentum at $\boldsymbol{\theta}^{+}$and $\boldsymbol{\theta}^{-}$, respectively.

213 The above method enables the flexible escape of U-Turn, but the arbitrary stop of the

214 leapfrog breaks the time reversibility. The NUTS algorithm overcomes this issue to

215 introduce a procedure such as the slice sampling, one of the MCMC methods; this is done

216 by exploring from the current point $\boldsymbol{\theta}^{(\tau)}$ by building a binary tree to a random time

217 direction (a sign of e), selecting the candidate state set $C$ based on the condition below,

218 and eventually selecting the subsequent states from $C$ uniformly.

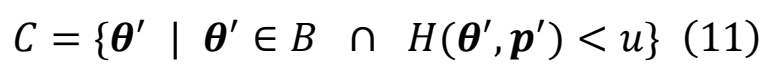

221

222 where $B$ is the set of all the visited state and $u$ is the so-called slice generated as a

223 uniform random number between 0 and the current posterior probability

$224 \exp \left(-H\left(\boldsymbol{\theta}^{(\tau)}, \boldsymbol{p}^{(\tau)}\right)\right)$ 


\subsubsection{Comparison with Metropolis-Hastings}

226 To verify the accuracy and efficiency of our method, we used the random-walk M-H

227 that was adopted by Ohno et al. (2021) to compare the estimation by the HMC method to

228 that by the M-H method (Metropolis et al. 1953; Hastings 1970), which is often used to

229 solve the geophysical problem (Fukuda and Johnson 2008; Ito et al. 2012; Dettmer et al.

230 2014; Minson et al. 2014; Ohno et al. 2022). Although Ohno et al. (2021) introduced an

231 automatic determination method for likelihood variance and parallel tempering, we use

232 the same variance value as HMC and estimate using only a single Markov chain for a

233 single parameter to compare both methods strictly. Another part of M-H conforms to that

234 of Ohno et al. (2021).

$235 \quad 2.3$ Settings of a single rectangular fault estimation

236 We estimated parameter $\boldsymbol{\theta}$ of the single rectangular fault model in Okada (1992) from

237 permanent displacement data d based on real-time GNSS by the HMC and NUTS

238 algorithms. In this section, we describe the setting of the estimation, such as the prior

239 distribution or likelihood function. 
241 We prepare a normal distribution as a prior of latitude and longitude and a uniform

242 distribution as a prior of others. We also set a uniform distribution, which is assumed to

243 exclude the fault that has an extreme stress drop value, for the stress drop and the ratio of

244 the fault length and width, which is assumed to have a shorter length than its width, to

245 improve the efficiency of exploration. Table 1 shows all prior distributions.

$$
\boldsymbol{r}_{i}=\widehat{\boldsymbol{d}}_{l}(\boldsymbol{\theta})-\mathbf{d}_{i}(13)
$$

253 where $\mathrm{N}$ is the number of stations, and $\widehat{\boldsymbol{d}}_{l}(\boldsymbol{\theta})$ is the surface displacement of model $\boldsymbol{\theta}$

254 calculated by the method of Okada (1992). Subscripts $\mathrm{h}$ and $\mathrm{v}$ represent the 
that they were independent. Equation (12) is based on the assumption that the residuals,

257 including the model and observational error, follow the normal distribution with mean 0

258 and variance $\sigma_{i}^{2}$.

\subsubsection{Change in variables}

HMC and NUTS use a gradient of posterior PDF to transition parameters. We observed

262 the gradients of latitude and longitude to be significantly large, except for seven

263 parameters, resulting in the very low efficiency of the exploration. A potential method for

264 correcting the gradients is the application of an adjustment hyperparameter, e, which is

265 the coefficient of the gradient, but parameter e is common; therefore, addressing our

case is complicated by the differences among each gradient. Therefore, we applied a

change of variables to the seven parameters to change the parameter scale. In addition,

certain previous tests have shown that a diminution in exploration efficiency can occur in 
were applied to logit (Equation (15)).

$273 \log$ translation from $x \in(a, \infty)$ to $x^{\prime} \in(-\infty, \infty)$ :

274

$$
x^{\prime}=\log (x-a)(14)
$$

275 logit translation from $x \in(a, b)$ to $x^{\prime} \in(-\infty, \infty)$ :

$$
x^{\prime}=\operatorname{logit}(x)=\log \left(\frac{x-a}{b-x}\right)
$$

Equations (14) and (15), for example, Depth $\in(0, \infty)$ is translated to Depth' $=$ $\log ($ Depth $) \quad$ and Strike $\in(0,360) \quad$ to Strike $^{\prime}=\log \{$ Strike $/(360-$ Strike $)\}$.

$$
f(\boldsymbol{\theta} \mid \mathbf{d})=f(\boldsymbol{\theta}) \times f(\mathbf{d} \mid \boldsymbol{\theta}) \times|\mathbf{J}|(16)
$$

$$
|\mathbf{J}|=e^{\text {Depth }^{\prime}} e^{\text {Length }^{\prime}} e^{\text {Width }^{\prime}} e^{\text {Slip }^{\prime}} \frac{360 e^{\text {Strike }^{\prime}}}{\left(1+e^{\text {Strike }}\right)^{2}} \frac{90 e^{\text {Dip }^{\prime}}}{\left(1+e^{\text {Dip }}\right)^{2}} \frac{360 e^{\text {Rake }}}{\left(1+e^{\text {Rake }}\right)^{2}}
$$


288 Note that we set the parameter's domain as Strike $\in(0,360)\left[{ }^{\circ}\right]$, Dip $\in(0,90)\left[^{\circ}\right]$, 289 and Rake $\in(-180,180)\left[^{\circ}\right]$, respectively.

\subsection{Convergence test}

292 In this study, the convergence of each Markov chain was evaluated using two criteria.

293 First, we confirmed the smoothness of the histograms for each model parameter. Although

294 it is a subjective perspective, it is required for convenience in later discussions using

295 statistics such as mode. Second, we utilized the method of convergence decision by

296 Gelman (1996) for objective judgment. This method can be used to determine the

297 convergence by comparing a chain's variance between a full chain and a divided one. We applied this test to all model parameters individually and confirmed their convergence.

299 If the Markov chain whose length is $\mathrm{T}$ excluded burn-in, it is divided into $\mathrm{K}$ equal 300 chains, and $\mathrm{T} / \mathrm{K}$ length chains are prepared. Then, the following two indices of a chain's variance are calculated, where $\bar{\theta}$ and $\bar{\theta}_{k}(k=1,2, \ldots K)$ are the mean values of a parameter in the full chain and the $k$ th divided chain, respectively. 


$$
\mathrm{VAR}_{\mathrm{B}}=\frac{\mathrm{T}}{\mathrm{K}-1} \sum_{k=1}^{\mathrm{K}}\left(\bar{\theta}_{k}-\bar{\theta}\right)^{2}
$$

305

$$
\mathrm{VAR}_{\mathrm{W}}=\frac{1}{\mathrm{~K}(\mathrm{~T}-1)} \sum_{k=1}^{\mathrm{K}} \sum_{t=1}^{\mathrm{T}}\left(\theta_{k}^{(t)}-\bar{\theta}_{k}\right)^{2}
$$

306

307 Equations (18) and (19) roughly represent the variance among the chains and the average 308 variance of each chain, respectively. Then, the index of a convergence decision using the two indices above is expressed as follows:

$$
R=\sqrt{\frac{\mathrm{T}-1}{\mathrm{~T}}+\frac{1}{\mathrm{~T}} \frac{\mathrm{VAR}_{\mathrm{B}}}{\mathrm{VAR}_{\mathrm{W}}}}(20)
$$

313 The $R$ value is almost 1 on the converged chain; in fact, limitless sampling may yield a converged Markov chain; it is explicit for Equation (20) that the $R$ value converges to

3151 , where $\mathrm{T} \rightarrow \infty$. In this study, the threshold of convergence is given as $R<1.1$, according to Gelman (1996). 


\subsection{Data and the setting of hyperparameters}

319 We applied our method to the 2016 Kumamoto earthquake (Mw 7.0), which occurred at

including three components (east-west, north-south, and up-down) were obtained using the REGARD system (Kawamoto et al. 2016) in real-time, and we used the data at $60 \mathrm{~s}$ after the mainshock at 200 stations near the source (Figure 2). algorithm explained above. In contrast, the step size of leapfrog $e$ was determined using trial and error. The length of the entire chain was determined using the convergence test explained above, and the length of the burn-in chain was determined by evaluating the trace plot of parameters, especially variance reduction (VR). Figures S1 and S2 show the results of the convergence test and the entire trace plot of each parameter, respectively. 
334 Amey et al. 2018); therefore, we also removed the first $5 \%$ of the chain similarly.

335 Although the observation error of GNSS may appear to result from the horizontal error

336 being smaller than the vertical error, we assumed the standard deviation of likelihood, $\sigma_{\mathrm{h}}$

337 and $\sigma_{\mathrm{v}}$, representing the same error value, $\sigma_{\mathrm{h}}=\sigma_{\mathrm{v}}=2 \mathrm{~cm}$ to simplify the inversion.

338 Table 2 also shows the hyperparameters in terms of M-H sampling. The entire burn-in

339 chain length was determined in the same way as the HMC, a factor of 50, compared to

340 HMC (Figure S1, S2). Note that the M-H method requires other hyperparameters and the

341 maximum transition size for each parameter.

342 


\section{Results}

\subsection{Expression of posterior probability density functions}

345 For geophysical interpretation, the posterior PDFs regarding the fault parameters are

346 required. However, we can obtain the rescaled PDFs directly using this method because

347 of the change in variables (detailed in 2.3.3). Moreover, the application of log and logit

348 projection, whose Jacobian matrix is complex, for unknown parameters complicates the

349 restoration of their scale. Therefore, we suggest special plotting for log and logit

350 translation that maintains the frequency of each class and inversely changes its upper and

351 lower limits. Then, we can obtain the expected PDFs that represent the correct posterior

352 probability and each parameter scale. The change in appearance following the use of our

353 expression method is shown in Figure S3.

\subsection{Results of the application for Kumamoto earthquake}

Figure 3 shows the posterior PDFs of each unknown parameter (latitude, longitude, 
360 The results of HMC in Figure 3 denote a shallow right-lateral fault dipping toward the

361 northwest, and the displacement predictions calculated from each sample are consistent

362 with the data (VR $88 \%$ ). The distribution of $\mathrm{Mw}$ is a reasonable estimation with a peak

363 of approximately 7.0, which is consistent with previous studies (Asano and Iwata 2016;

364 Kawamoto et al. 2016; Yarai et al. 2016; Tanaka et al. 2019).

365 Figure 3 shows considerable features that PDFs obtained using HMC accord with by M-

$366 \mathrm{H}$ in the shape of PDFs or statistics, nevertheless, both methods estimate independently.

367 This means that HMC can be applied to the single rectangular fault estimation.

368 Conversely, there are a few differences in PDF of depth, it can affect by log translation,

369 which implicitly assumes a non-zero for depth. Its width is extremely narrow, and its

statistics are approximately 0 . The estimation of VR using HMC is also similar to that using $\mathrm{M}-\mathrm{H}$. Therefore, the non-zero assumption with $\log$ translation seems to be

sufficiently available for a practical application.

373 For a comparison of the surface deformation, Figure 4 (a) shows the rectangular fault of

374 a mode model (the so-called maximum a posteriori model) solved by HMC, and the 
375 predicted displacement at each station assumed the model with the displacement data we

376 used. Figure 4 also shows a mode model solved by M-H. As already mentioned, the HMC

377 mode model adequately explains the displacement data and is consistent with the M-H

378 model. As illustrated in figure 4 (b), which is drawn using a method proposed by Ohno et

379 al. (2021), we exhibited the spatial frequency of the fault rectangular. This figure includes

380 the color scale considering overlaps among the rectangles of each HMC sample on the

381 map. The HMC models likely contain only a small uncertainty of estimation because the

382 color scale expands in a significantly narrow region. 


\section{Discussion}

\subsection{Comparison of initial exploration}

Figure 5 shows the Markov chains of the first 1,000 samples in the HMC and M-H

methods. The exploration of HMC can produce estimates that are approximately the same as the final products within 40 chains and a VR of $88 \%$. In contrast, the trace plots of M-

case comparing both the methods, our results are important in being able to ensure

efficient convergence of $\mathrm{HMC}$, where $\mathrm{HMC}$ required less than $1 \%$ chains of M-H's for model exploration. To fully utilize this efficiency, this method should be applied to high-

dimensional problems such as slip distribution estimation or inversion using non-linear

Green's function, which incurs a high computational cost in one step.

efficiency. The rake angle or slip amount chains using the $\mathrm{M}-\mathrm{H}$ method initially move in 
method because of the transition using gradients of the posterior PDF. The chains of

401 latitude or strike denote that even if HMC passes by following the final statistics, a quick

402 return to the same value is expected. It is an interesting exploration tendency showing the

403 ability of the HMC method to explore a wide range of parameters in one step and aim at

404 a minimum posterior immediately. These features cannot be realized by the M-H method,

405 whose transition is performed by adding only a small amount and whose acceptance ratio

406 is generally low.

407 Figure 6 shows the marginal PDFs appearing in pairs of parameters fault length, fault

408 width and slip amount. The figures using all parameters are shown in Figure S4-S7.

409 Figures $6 \mathrm{a}(\mathrm{HMC})$ and $6 \mathrm{~b}(\mathrm{M}-\mathrm{H})$ display the marginal PDFs including the burn-in chains,

410 indicating that the HMC method can reach the high probability area effectively with

411 straight and stepping trace. This method only trace in the plausible area because of the

412 larger white area, which has never been explored, rather than the M-H method.

413 Considering that Figures 6c (HMC) and 6d (M-H) show that the HMC method can

414 generate a similar correlation to the M-H among parameters, and we confirmed the lean

415 sampling of the HMC method. 
416 However, the HMC or other MCMC methods have the problem of ensuring minimum

417 global exploration. One of the methods to certainly and efficiently explore more broadly

418 in parameter space is parallel tempering (e.g., Dosso et al. 2012; Sambridge 2013;

419 Dettmer et al. 2014; Hallo and Gallovič 2020; Ohno et al. 2021,2022). In future work, we

420 will develop a method that introduces this expanding method to the HMC and realize a

421 more efficient and stable fault estimation.

422

$423 \quad 4.2$ Comparison regarding the autocorrelation of Markov chains

424 As mentioned previously, the $\mathrm{M}-\mathrm{H}$ method conducts the sampling of parameter

425 exploration by slightly perturbating model parameters. In contrast, HMC transitions from

426 place to place and visits plausible samples if they are far away. Given these sampling

427 characteristics, we analyzed the autocorrelation of Markov chains generated by both

428 methods.

429 Figure 7 shows the power spectral densities of the Markov chain regarded as a time

430 series and its inclination ("a" value in the figure) using log approximation. In this figure,

431 the results of the $\mathrm{M}-\mathrm{H}$ method explicitly denote the inverse proportion to frequency 
$432 f[$ /count $]$ and show random walk tendency; the inclination is roughly $f^{-2}$. The 433 inclination in the zone over 1,000 chains appears to be flat, similar to white noise.

434 Conversely, the results of the HMC method denote an inclination of less than 0.5 , and a

435 white noise-like tendency for almost all parameters. Only the result of the depth parameter

436 denotes a larger inclination than the HMC's other results, nearly 1.5, although it is slightly

437 smaller than that of the M-H method. This high autocorrelation is considered to be caused

438 by the exploration near the domain. Hallo and Gallovič (2020) address similar problems

439 using the unique prior PDF that is reflected in the parameter domain.

440 Generally, for MCMC sampling, a higher autocorrelation tends to induce deterioration

441 of sampling efficiency and complicates the achievement of the proper posterior PDFs by

442 finite-length chains (Geyer 2011). As discussed above, M-H sampling is often highly

443 correlated between close samples. The thinning method, which discards all but every $k$ th

444 sampled value, is likely to address this problem (Link and Eaton 2012), but it does not

445 guarantee improved estimation efficiency (Geyer 2011; Link and Eaton 2012). In addition,

446 even if we adopt this method to introduce to $\mathrm{M}-\mathrm{H}$, we must abandon several thousand

447 samples for one sample because the PSD analysis shows a high autocorrelation in the 
448 zone over 1,000 chains. Therefore, it is conceivable that the HMC method can efficiently

449 achieve lower autocorrelation sampling without wasting samples.

450

$451 \quad 4.3$ More optimized utilization of the HMC

452 As discussed above, at the point of the number of chains, compared to the M-H method,

453 the HMC method can estimate posterior PDFs more efficiently in the Bayesian analysis

454 of seismic fault estimation. However, the HMC can also be less efficient at the point of

455 the calculation time in general, especially in the case of applying to the problem where

456 one chain's computational cost of the forward calculation is low, such as in our problem.

457 To reduce the calculation time, a method for optimizing hyperparameter e, which

458 represents the step size of the leapfrog, should be developed. For example, the Stan

459 algorithm (Hoffman and Gelman 2014) employs the dual averaging method developed

460 by Nesterov (2009), a stochastic gradient descent method, which is expected to optimize

461 in the same way as our method does.

462 In addition to the hyperparameter, the calculation time of the estimation relies on the

463 number of model parameters. It is difficult for the random walk M-H method to conduct 
464 efficient sampling in a parameter space that consists of many parameters because the

465 space is vast and ambiguous for model exploration (Roberts 1998, Ringer 2021).

466 However, as reported by Fichtner and Simute (2018), the HMC method can maintain the

467 calculation time because of the differentiation of the posterior PDF, which contributes to

468 ensuring the transition to the high posterior probability region. Therefore, the HMC can

469 be superior and play an important role in solving high-dimensional problems such as slip

470 distribution estimation or problems that require copious amounts of resources for forward

471 computation.

472 
474 We developed a fault estimation algorithm using the HMC method; this was the first

475 attempt to use the HMC method for fault estimation. The developed method performs

476 efficiently following the adjustment of each parameter scale by changing the variables,

477 making good use of the log and logit functions. We then applied the developed method to

478 the 2016 Kumamoto earthquake $\left(\mathrm{M}_{\mathrm{j}} 7.3\right)$ and assessed its feasibility for the single

479 rectangular fault estimation and its exploration efficiency through comparison with the

480 M-H method.

481 We found that the HMC method estimates the informative model that reproduces the

482 observation data and substantially corresponds to the fault model predicted by the M-H

483 method, although both algorithms perform the estimation independently. This indicates

484 that the HMC method can be utilized for the single rectangular fault estimation. The

485 length of the Markov chain required for the HMC sampling was approximately $2 \%$ of

486 that of the M-H method, and the $1 \%$ chains were needed to explore the high VR fault

487 models (VR 88 \%). We also clarified that the HMC method works as a low 
489 Markov chains of each parameter. These results are attributed to the theoretical 490 characteristics of the HMC method, which allows for efficient search following the 491 gradient of the posterior distribution. This reasonable HMC sampling appears to be valid 492 for high-dimensional problems such as slip distribution estimation or problems using 493 nonlinear Green's function, which incurs high computational costs for one chain.

494 In contrast, the HMC method requires a longer calculation time for one chain than the 495 M-H method. This indicates that the use of the M-H method is more suitable than that of 496 the HMC for single rectangular fault estimation, a low-dimensional problem. Therefore, 497 the choice between the M-H or HMC methods depends on the cost of forwarding 498 calculation and the number of unknown parameters in the applied problem. 499 


\section{Declarations}

\section{List of abbreviations}

502

HMC: Hamiltonian Monte Carlo

503

504

505

506

507

508

509

510

PSD: Power Spectral Density

PDF: Probability Density Function

M-H: Metropolis-Hastings

MCMC: Markov Chain Monte Carlo

REGARD: REal-time GEONET Analysis system for Rapid Deformation

monitoring

GNSS: Global Navigation Satellite System

VR: Variance Reduction

512

Availability of data and materials

513

514

515
All 30-s GEONET raw data for post-processing can be obtained via the GSI

website. Other data used and/or analyzed in this study are available from the

corresponding author upon reasonable request. 


\section{Competing interests}

The authors declare that they have no competing interests.

\section{Funding} 21H05001). 
This work was supported by the JST FOREST Program (grant number: JPMJFR202P, Japan). This study was also supported by the Ministry of 21H05001). \\ 546 References}

547 Agata R, Kasahara A, Yagi Y (2021) A Bayesian inference framework for fault slip 
Amey R, Hooper A, Walters R (2018) Bayesian method for incorporating self-similarity into earthquake slip inversions. J Geophys Res: Solid Earth 123:6052-6071. doi: 10.1029/2017JB015316

Asano K, Iwata T (2016) Source rupture processes of the foreshock and mainshock in the 2016 Kumamoto earthquake sequence estimated from the kinematic waveform inversion of strong motion data. Earth Planets Space 68:1-11. doi: fault inversion. Geophys J Int 199:735-751. doi: 10.1093/gji/ggu280

Dosso SE, Holland CW, Sambridge M. (2012) Parallel tempering for strongly nonlinear geoacoustic inversion. J Acoust Soc Am 132:3030-3040. doi: $10.1121 / 1.4757639$ 
Duputel Z, Agram PS, Simons M, Minson SE, Beck JL. (2014) Accounting for 197:464-482. doi: 10.1093/gji/ggt517

Dutta R, Jónsson S, Vasyura-Bathke H. (2021) Simultaneous Bayesian Estimation of Earth 126:1-28. doi: 10.1029/2020JB020441 10.1002/2017JB015249

574 Fichtner A, Zunino A, Gebraad L (2019) Hamiltonian Monte Carlo solution of tomographic inverse problems. Geophys J Int 216:1344-1363. doi: 10.1093/gji/ggy496

577 Fukahata Y, Wright TJ. (2008) A non-linear geodetic data inversion using ABIC for slip 
580

581

582

583

584

585

586

587

588

589

590

591

592

593

594

595

Fukuda J, Johnson K (2008) A fully Bayesian inversion for spatial distribution of fault slip with objective smoothing. Bull Seismol Soc Am 98:1128-1146. doi: $10.1785 / 0120070194$

Fukuda J, Johnson K (2010) Mixed linear-non-linear inversion of crustal deformation data: Bayesian inference of model, weighting and regularization parameters. Geophys J Int 181:1441-1458. doi: 10.1111/j.1365-246X.2010.04564.x

Gebraad L, Boehm C, Fichtner A (2020) Bayesian Elastic Full-Waveform Inversion Using Hamiltonian Monte Carlo. J Geophys Res: Solid Earth 125:1-18. doi: 10.1029/2019JB018428

Gelman A. (1996) Inference and Monitoring Convergence. In: Markov Chain Monte Carlo in Practice. Chapman \& Hall/CRC, London

Gelman A, Carlin J, Stern H, Dunson D, Vehtari A, Rubin D. (2021) Bayesian Data Analysis Third edition (with errors fixed as of 15 February 2021). Chapman \& Hall/CRC, London

Geyer CJ. (1991) Markov Chain Monte Carlo Maximum Likelihood. In Computing Science and Statistics, Proceedings of the 23rd Symposium on the Interface, 
597 Geyer CJ. (2011) Introduction to Markov Chain Monte Carlo. In: Handbook of Markov

Hallo M, Gallovič F (2020) Bayesian Self-Adapting Fault Slip Inversion with Green's Functions Uncertainty and Application on the 2016 Mw7.1 Kumamoto Earthquake. J Geophys Res: Solid Earth 125:1-32. doi: 10.1029/2019JB018703

Hastings W (1970) Monte Carlo sampling methods using Markov chains and their applications. Biometrika 57:97-109. doi: 10.1093/biomet/57.1.97 in Hamiltonian Monte Carlo. J Mach Learn Res 15:1593-1623. 
613 Kawamoto S, Ohta Y, Hiyama Y, Todoriki M, Nishimura T, Furuya T, Sato Y, Yahagi T, modeling system for GEONET. J Geophys Res: Solid Earth 122:1324-1349. doi: 10.1002/2016JB013485

Link WA, Eaton MJ (2012) On thinning of chains in MCMC. Methods Ecol Evol 3:112115. doi: 10.1111/j.2041-210X.2011.00131.x

Mai PM, Beroza GC (2002) A spatial random field model to characterize complexity in earthquake slip. J Geophys Res: Solid Earth 107:ESE 10-1-ESE 10-2. doi: 10.1029/2001JB000588 state calculations by fast computing machines. J Chem Phys 21:1087-1092. doi: $10.1063 / 1.1699114$ 
628

629

630

631

632

633

634

635

636

637

638

639

640

641

642

643

Muir JB, Tkalčić H (2020) Probabilistic lowermost mantle P-wave tomography from hierarchical Hamiltonian Monte Carlo and model parametrization crossvalidation. Geophys J Int 223:1630-1643. doi: 10.1093/gji/ggaa397

Musa A, Watanabe O, Matsuoka H, Hokari H, Inoue T, Murashima Y, Ohta Y, Hino R, Koshimura S, Kobayashi H (2018) Real-time tsunami inundation forecast system for tsunami disaster prevention and mitigation. J Supercomput 74:30933113. doi: $10.1007 / \mathrm{s} 11227-018-2363-0$

Neal RM. (2011) MCMC using hamiltonian dynamics. In: Handbook of Markov Chain Monte Carlo. Chapman \& Hall/CRC, London

Nesterov Y (2009) Primal-dual subgradient methods for convex problems. Math Program 120:221-259. doi: 10.1007/s10107-007-0149-x

Ohno K, Ohta Y, Kawamoto S, Abe S, Hino R, Koshimura S, Musa A, Kobayashi, H. (2021) Real-time automatic uncertainty estimation of coseismic single rectangular fault model using GNSS data. Earth Planets Space 73:127. doi: $10.1186 / \mathrm{s} 40623-021-01425-0$

Ohno K, Ohta Y, Hino R, Koshimura S, Musa A, Abe T, Kobayashi H (2022) Rapid and 
Ohta Y, Ohzono M, Miura S, Iinuma T, Tachibana K, Takatsuka K, Miyao K, Sato T, Res: Solid Earth 117:1-16. doi: 10.1029/2011JB008750

657 Ohta Y, Inoue T, Koshimura S, Kawamoto S, Hino R. (2018) Role of real-time GNSS in 658 near-field tsunami forecasting. J Disaster Res 13:453-459. doi:

659 10.20965/jdr.2018.p0453 
660 Okada Y. (1992) Internal deformation due to shear and tensile faults in a half-space. B Seismol Soc Am 82:1018-1040.

662

663

664

665

666

667

668

669

670

671

672

673

674

675

Ringer H, Whitehead JP, Krometis J, Harris RA, Glatt-Holtz N, Giddens S, Ashcraft C, Carver G, Robertson A, Harward M, Fullwood J, Lightheart K, Hilton R, Avery A, Kesler C, Morrise M, Klein MH (2021) Methodological Reconstruction of Historical Seismic Events From Anecdotal Accounts of Destructive Tsunamis: A Case Study for the Great 1852 Banda Arc Mega-Thrust Earthquake and Tsunami. J Geophys Res: Solid Earth 126:1-23. doi: 10.1029/2020JB021107

Roberts GO, Rosenthal JS (1998) Optimal scaling of discrete approximations to Langevin diffusions. J R Stat Soc Ser B Methodol 60:255-268. doi: $10.1111 / 1467-9868.00123$

Sambridge M, Mosegaard K. (2002) Monte Carlo methods in geophysical inverse problems. Rev Geophys 40:3-1-3-29. doi: 10.1029/2000RG000089

Sambridge M (2013) A parallel tempering algorithm for probabilistic sampling and multimodal optimization. Geophys J Int 196:357-374. doi: 10.1093/gji/ggt342 Swendsen RH, Wang JS. (1986) Replica Monte Carlo simulation of spin-glasses. Phys 
677 Tanaka Y, Ohta Y, Miyazaki S. (2019) Real-Time Coseismic Slip Estimation via the

678

679

680

681

682

683

684

685

686

687

688

689

690

691
GNSS Carrier Phase to Fault Slip Approach: A Case Study of the 2016

Kumamoto Earthquake. Geophys Res Lett 46:1367-1374. doi:

10.1029/2018GL080741

Tarantola A. (2005) Inverse problem theory and Methods for Model Parameter

Estimation. Society for Industrial and Applied Mathematics.

Yarai H, Kobayashi T, Morishita Y, Fujiwara S, Hiyama Y, Kawamoto S, Ueshiba H, Miura Y, Miyahara B. (2016) Source fault models of the 2016 Kumamoto earthquake inverted from crustal deformation. Bull of the Geospatial Information Authority of Japan 128:169-176. (in Japanese)

\section{Table Legends}

Table 1. Prior parameter for fault parameters and certain calculated parameters. A mean

of normal distribution for latitude and longitude is assumed as their initial parameter. A reference point of latitude and longitude is a fault center, and depth is fault top. $\mathrm{N}\left(\mu, \sigma^{2}\right)$, 
represents a normal distribution with mean $\mu$, variance $\sigma^{2}$ and $\mathrm{U}(a, b)$ represents a uniform distribution with a lower limit $a$ and upper limit $b$.

694

695 Table 2. Setting of hyperparameters.

696

697

698

\section{Figure Legends}

699 Figure 1. Example of sampling using the Hamiltonian Monte Carlo (HMC) method. The contours show the constant Hamiltonian and their values. Triangles, circles, and squares show start points, transition points, and endpoints of each leapfrog transition, respectively. The blue triangle particularly represents the initial point of the sampling. In addition, the orange line shows the jump to the next momentum generated by standard normal distribution after acceptance of the candidate point.

706 Figure 2. Location of stations. A bigger map shows the red rectangular area in a smaller 
estimation.

709

710 Figure 3. Inferred posterior probability density function (PDF) due to Hamiltonian Monte

711 Carlo (HMC) and Metropolis-Hastings (M-H) estimation. Both results of HMC and M-

$712 \mathrm{H}$ are shown by blue and red lines, respectively. Each y-axis represents the frequency of

713 Markov chain Monte Carlo (MCMC) samples written by the same color as histograms.

714 Then the insert values represent statistics (the mean, median, mode, and length of $95 \%$

715 confidence interval of PDFs from top to bottom) similarly presented in blue or red.

716

717 Figure 4. Map view results. (a) Each rectangular denote the mode model; the red and

718 blue ones filled with slanted lines are inferred using $\mathrm{M}-\mathrm{H}$ and $\mathrm{HMC}$, respectively. In this

719 figure, gray lines show known active faults; black and white arrows show the data and

720 the mode model's horizontal displacements, respectively. Residuals between the data and

721 the model's vertical displacements are also shown in a color scale. (b) The frequency of

722 fault rectangular drawn using each HMC sample. The color scale represents the frequency,

723 whose warm color denotes a region of high frequency, and the region that is not colored 
indicates no estimation.

725

726 Figure 5. Trace plots of the first 1,000 samples. The chains of nine model parameters

727 (latitude, longitude, depth, strike, dip, rake angle, fault length, fault width, and slip

728 amount) and three calculated parameters (Mw, stress drop, and VR) are shown in each

729 figure. The blue and red solid lines indicate the traces produced by HMC and M-H,

730 respectively. Note that the black broken lines indicate the mode value of full chains

731 produced by HMC.

733 Figure 6. Two dimensional (2D)-marginal posterior PDFs of the HMC and M-H sampling.

734 Each figure indicates the correlation among part of the fault parameters (fault length, fault

width and slip amount). Upper figures show the marginal PDFs via the HMC (a) and M-

$\mathrm{H}$ (b) including burn-in exploration whose starting point is indicated by red star. Lowers

739 Figure 7. Power spectral density (PSD) of Markov chains of nine model parameters. In 
740 each figure, red and blue show the results of $\mathrm{M}-\mathrm{H}$ and $\mathrm{HMC}$, respectively. The light color

741 line shows PDF and the heavy color line shows the moving average of PDF (the average

742 of 25 points on the right and left side). The result of the log approximation is visually

743 shown by the straight line and its estimated inclination is inserted on the bottom left of

744 each figure(upper red: M-H, lower blue: HMC). 
Figures

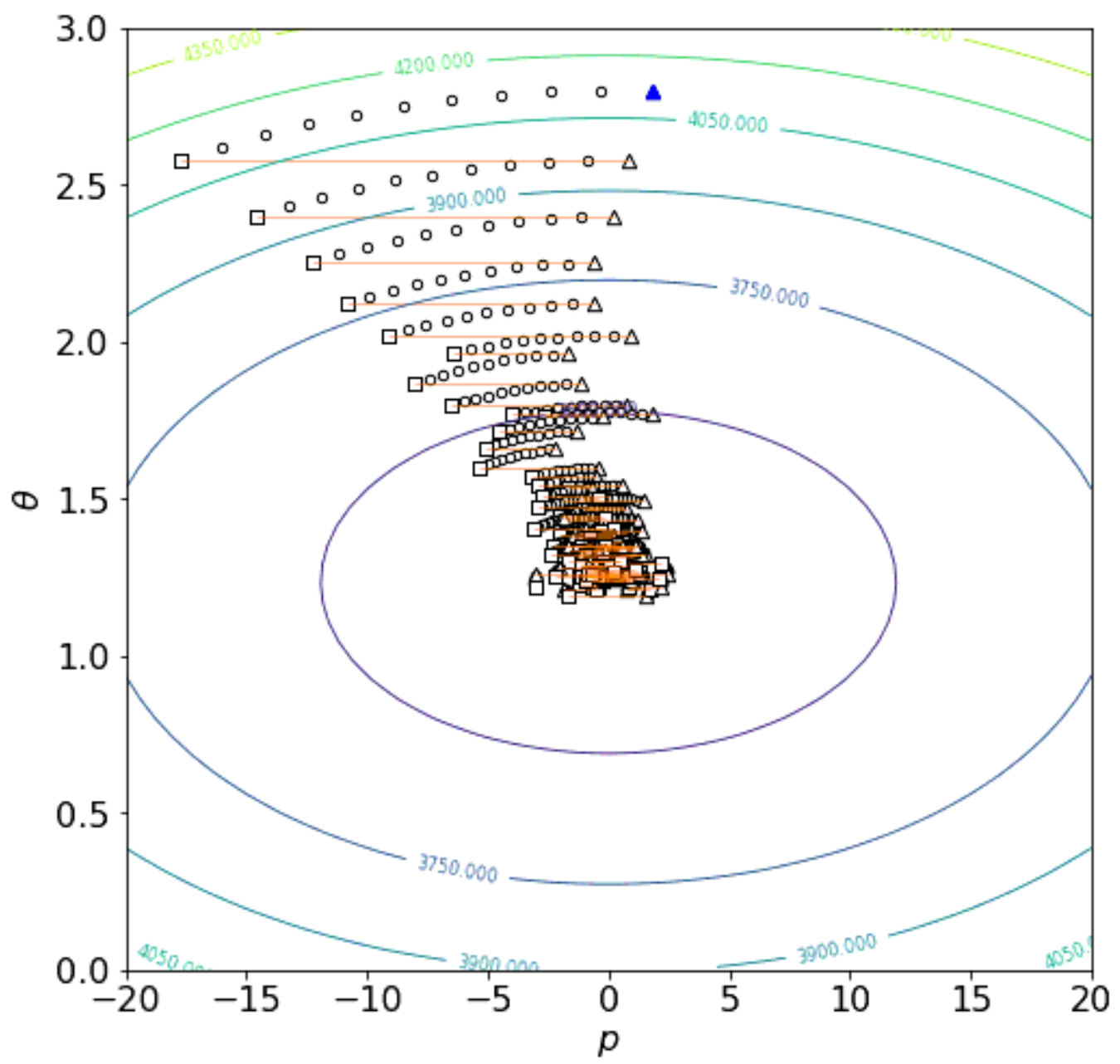

Figure 1

Example of sampling using the Hamiltonian Monte Carlo (HMC) method. The contours show the constant Hamiltonian and their values. Triangles, circles, and squares show start points, transition points, and endpoints of each leapfrog transition, respectively. The blue triangle particularly represents the initial point of the sampling. In addition, the orange line shows the jump to the next momentum generated by standard normal distribution after acceptance of the candidate point. 


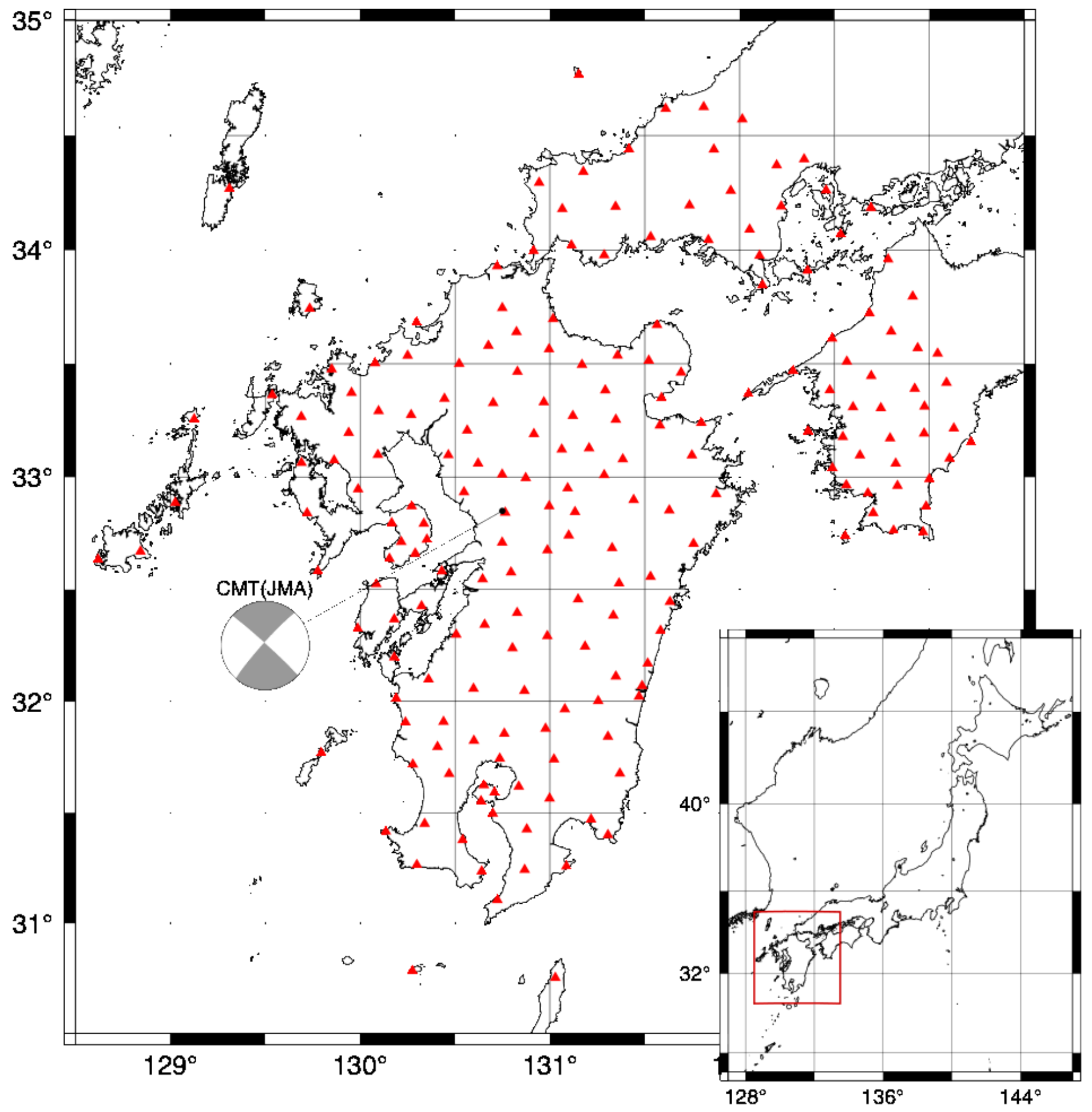

Figure 2

Location of stations. A bigger map shows the red rectangular area in a smaller one depicting the whole of Japan. Red triangles represent the 200 GNSS stations for estimation.

Figure 3 
Inferred posterior probability density function (PDF) due to Hamiltonian Monte Carlo (HMC) and Metropolis-Hastings $(\mathrm{M}-\mathrm{H})$ estimation. Both results of $\mathrm{HMC}$ and $\mathrm{M}-\mathrm{H}$ are shown by blue and red lines, respectively. Each y-axis represents the frequency of Markov chain Monte Carlo (MCMC) samples written by the same color as histograms. Then the insert values represent statistics (the mean, median, mode, and length of $95 \%$ confidence interval of PDFs from top to bottom) similarly presented in blue or red.
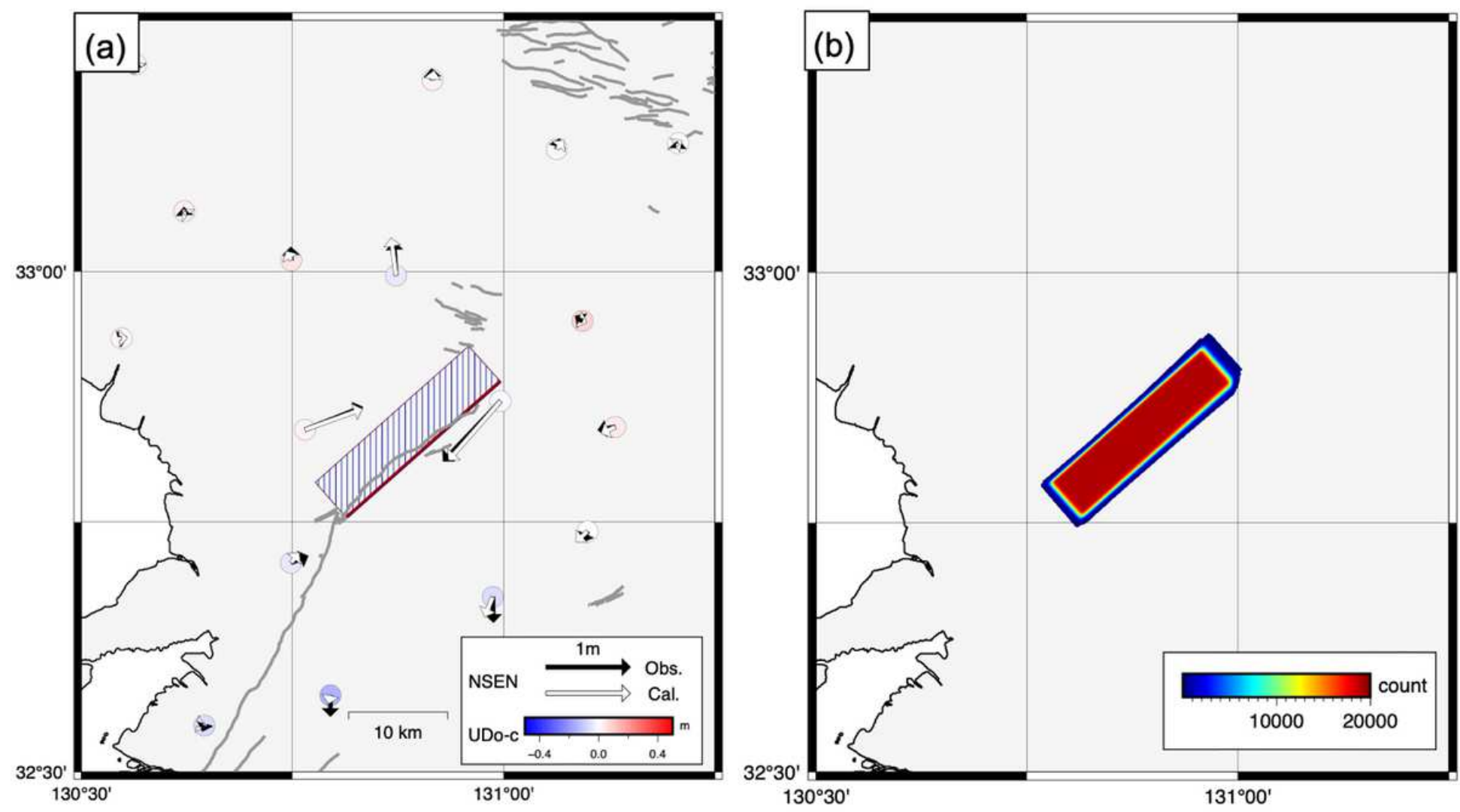

Figure 4

Map view results. (a) Each rectangular denote the mode model; the red and blue ones filled with slanted lines are inferred using $\mathrm{M}-\mathrm{H}$ and $\mathrm{HMC}$, respectively. In this figure, gray lines show known active faults; black and white arrows show the data and the mode model's horizontal displacements, respectively. Residuals between the data and the model's vertical displacements are also shown in a color scale. (b) The frequency of fault rectangular drawn using each HMC sample. The color scale represents the frequency, whose warm color denotes a region of high frequency, and the region that is not colored indicates no estimation.

\section{Figure 5}

Trace plots of the first 1,000 samples. The chains of nine model parameters (latitude, longitude, depth, strike, dip, rake angle, fault length, fault width, and slip amount) and three calculated parameters ( $\mathrm{Mw}$, stress drop, and VR) are shown in each figure. The blue and red solid lines indicate the traces produced 
by $\mathrm{HMC}$ and $\mathrm{M}-\mathrm{H}$, respectively. Note that the black broken lines indicate the mode value of full chains produced by HMC.

\section{Figure 6}

Two dimensional (2D)-marginal posterior PDFs of the $\mathrm{HMC}$ and $\mathrm{M}-\mathrm{H}$ sampling. Each figure indicates the correlation among part of the fault parameters (fault length, fault width and slip amount). Upper figures show the marginal PDFs via the HMC (a) and $\mathrm{M}-\mathrm{H}(\mathrm{b})$ including burn-in exploration whose starting point is indicated by red star. Lowers also show the marginal PDFs via the HMC (c) and $\mathrm{M}-\mathrm{H}$ (d) but excluded burn-in samples.

\section{Figure 7}

Power spectral density (PSD) of Markov chains of nine model parameters. In each figure, red and blue show the results of $\mathrm{M}-\mathrm{H}$ and $\mathrm{HMC}$, respectively. The light color line shows PDF and the heavy color line shows the moving average of PDF (the average of 25 points on the right and left side). The result of the log approximation is visually shown by the straight line and its estimated inclination is inserted on the bottom left of each figure(upper red: $\mathrm{M}-\mathrm{H}$, lower blue: $\mathrm{HMC}$ ).

\section{Supplementary Files}

This is a list of supplementary files associated with this preprint. Click to download.

- GraphicalAbstract.png

- SupplementaryInformation.docx 